\title{
Grading nuclear cataract: reproducibility and validity of a new method
}

\author{
Nigel F Hall, Philip Lempert, Rosaleen P Shier, Rahila Zakir, David Phillips
}

\begin{abstract}
Aims-To assess the reproducibility and validity of a new instrument for grading nuclear cataract-the laser slit lamp, by comparison with an established method of lens grading - the Lens Opacities Classification System III (LOCS III).

Methods-62 volunteers (113 eyes) were examined on two occasions. At each visit, a video image of the anterior segment was captured with the laser slit lamp and stored digitally. A measure of lens opacity for each laser slit lamp image was calculated using image analysis software. Each lens was also photographed on both visits for LOCS III grading of nuclear colour and nuclear opalescence.

Results-There was a linear increasing relation between the laser slit lamp measure of nuclear lens opacity and the LOCS III nuclear opalescence scores. The overall reproducibility of the laser slit lamp measurement was comparable with LOCS III (intraclass correlation coefficient of 95\% (95\% CI 92.9-96.5) for the laser slit lamp method; 97\% (95\% CI 95.6-97.9) for the LOCS III method). However, among healthy subjects, the reproducibility was lower (intraclass correlation for the laser slit lamp of $38.6 \%$ (95\% CI 12.9-59.2) and $76.1 \%$ (95\% CI 62.3-85.4) for LOCS III.
\end{abstract}

Medical
Council

Environmental

Epidemiology Unit

(University of

Southampton),

Southampton General

Hospital,

Southampton

N F Hall

R P Shier

D Phillips

Department of Physiology, School of Veterinary Medicine, Cornell University, Ithaca, NY, USA

P Lempert

Southampton Eye Unit, Southampton General Hospital, Southampton R Zakir

Correspondence to: Dr Nigel F Hall, MRC Environmental Epidemiology Unit, (University of

Southampton), Southampton General Hospital, Tremona Road, Southampton SO16 6YD.

Accepted for publication 25 June 1999 assessment of cataract type and severity. These methods are designed to be applied by an oph- thalmologist who is required to grade the appearance of lenses under study against set pictorial standards. Good intraobserver agreement was demonstrated for nuclear cataract grading in these studies. ${ }^{34}$ Interobserver agreement tended to be lower, however, leading both reports to conclude that for longitudinal studies of cataract, both initial and follow up examinations should be performed by the same ophthalmologist. Furthermore, the coarseness of the scale used to assess nuclear cataract means that the methods may not be sensitive to small (but clinically or biologically significant) differences in nuclear cataract severity.

The Lens Opacities Classification System III

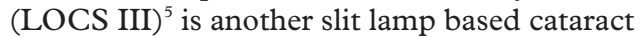
grading method. Lenses are graded for the three major types of cataract-nuclear, cortical, and posterior subcapsular. For each cataract type, the observer assigns a severity score to the lens, using the standard photographs. It may be applied clinically (direct slit lamp grading) or by photograding (grading of lens photographs taken using a standard protocol). The method allows the grader to interpolate between the supplied photographic standards thus allowing greater precision in estimating cataract severity. As with any primarily observer dependent system, LOCS III is vulnerable to inconsistencies in application of the system over time and between observers. This can be mitigated against by appropriate observer training but cannot be eliminated. Photograding is less subject to these effects than direct patient derived grading. However, as the devisers of LOCS III themselves point out "In the 'real world', the cost or inefficiency of photograding may be too high, especially when screening large numbers of patients rapidly in field conditions." ${ }^{5}$ The rationale of the more objective methods is to reduce the human component of the measurement as far as possible. Such a method is especially desirable in the case of nuclear cataract because it comprises the largest subgroup in many studies, ${ }^{67}$ and its diagnosis and grading is the most difficult. ${ }^{8}$

We have therefore developed a novel method of assessing the severity of nuclear cataract based on a standard slit lamp. The apparatus comprises an illumination arm that generates a slit of laser light, and a viewing arm with attached beam splitter and high sensitivity charge coupled device (CCD) camera allowing a video image of the laser illuminated anterior segment to be sent to a computer (Figs 1 and $2)$. The image is then analysed to measure the amount of light back scattered from the lens nucleus (Fig 3). The device was made using 


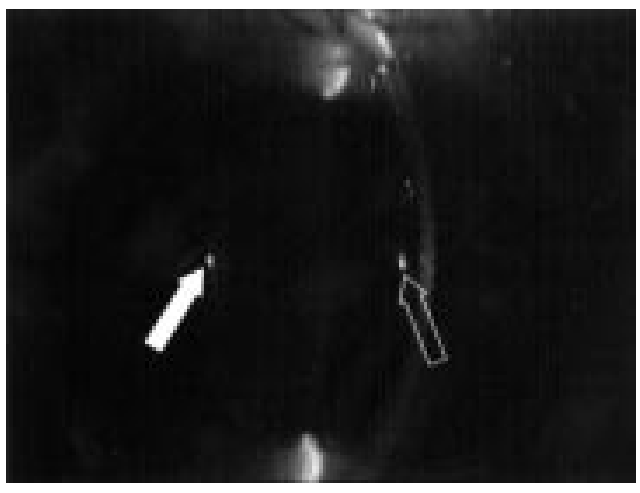

Figure 1 Digitised video image of the anterior segment of a normal subject taken with the laser slit lamp. The bright spots are Purkinje images I (open arrow) and IV (solid arrow) and were used to standardise alignment of the eye during image capture.

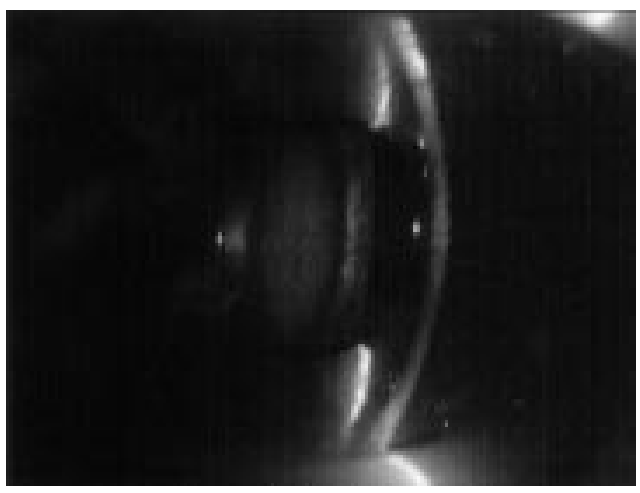

Figure 2 Laser slit lamp (digitised video image) view of the anterior segment of a subject with nuclear lens opacity.

readily available components. ${ }^{9}$ Its reproducibility and validity are unknown. We carried out a study to measure these variables as described below.

\section{Materials and methods}

Twenty nine volunteers from a research institution (six men and 23 women), aged between 20 and 61 years (mean 40.1 (SD 10.6)) took part in the study. None of them had a history of cataract. In addition, 33 volunteers (16 men and 17 women) from the cataract surgery waiting list at Southampton Eye Unit took part. They were aged between 64 and 90 years (mean $65.5(6.2)$ ). The aim was to apply the method across as wide as possible a range of nuclear lens opacity. Both eyes

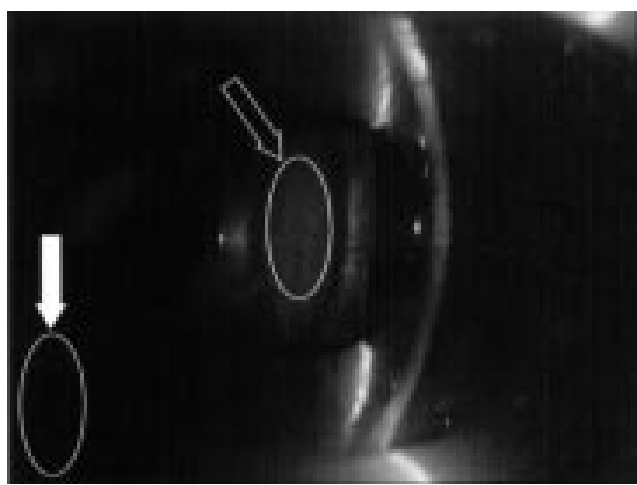

Figure 3 Laser slit lamp image showing how the region of interest mask (ellipse) was defined for the nuclear area (open arrow) and for the background area (solid arrow). were examined unless the fellow eye had had previous cataract surgery or the subject was only prepared to have one eye dilated. Thus, 113 eyes were imaged on two occasions.

Each participant attended for an assessment of lens opacity on two occasions. Participants' pupils were dilated before the measurements with tropicamide $1 \%$. One observer $(\mathrm{NH})$ carried out all image capture using the laser slit lamp, and performed all lens photography using a Zeiss anterior segment camera and Kodak Ektachrome 200ASA film. One subject was omitted from the analysis because the quality of the lens images was too poor to be satisfactorily analysed. During image capture, the lens was observed with a real time video "window" on the computer screen and the subjects' fixation controlled using the Purkinje images (Fig 1). Constant settings for the laser slit lamp's video camera and also for the anterior segment camera were maintained throughout the study. Two ophthalmologists $(\mathrm{NH}$, $\mathrm{RZ}$ ), individually graded the lens photographs (masked, and projected in random order) against LOCS III standards according to the stated protocol. ${ }^{5}$ In brief, LOCS III nuclear opalescence is graded by comparing the slide of the lens to be graded, with the standard nuclear images (standards 1-6). The grader then assigns a decimal grade to reflect the position of the unknown within the appropriate standard interval. The average opalescence of the entire nucleus in the lens being evaluated is compared with that in each of the standards. Similarly, nuclear colour is graded by comparing the colour of the lens to be graded with that in standard nuclear images 1-6 and using decimals to interpolate between the integer values of the reference standards. In this study, individual scores for each slide were then compared, and a consensus grade obtained by reference to the original slide.

The digital images from the laser slit lamp were analysed with IMAGE PRO PLUS software. ${ }^{10}$ To avoid bias during this procedure, all image files were randomly renamed before the analysis. A standard elliptical area of interest mask was defined to encompass as much of the lens nucleus as possible without including cortex. This was first estimated by trial and error with reference to a series of the anterior segment images. The dimensions of this estimated ellipse were then reduced slightly to take account of nuclei that might be smaller than those in the initial series. The standard elliptical mask thus obtained was then saved and used to define the area of interest in all subsequent analyses. A variable amount of outer nuclear shell would thus be excluded from the portion of the nucleus sampled using this method. This excluded area would tend to be larger in older subjects who generally have larger lens nuclei.

The mean pixel intensity within the mask (on a scale of 0 to 255) for the central nuclear region in each lens was calculated by the image analysis computer program. An "area of interest" mask with the same dimensions was then used to calculate the mean pixel intensity in a 
Table 1 Distribution of LOCS III nuclear colour and opalescence gradings in 113 eyes with gradable photographs according to subject type (healthy or from cataract waiting list)

\begin{tabular}{|c|c|c|c|c|}
\hline \multirow[b]{3}{*}{ LOCS III score } & \multicolumn{4}{|c|}{ Number of eyes (\%) } \\
\hline & \multicolumn{2}{|c|}{ Nuclear opalescence } & \multicolumn{2}{|c|}{ Nuclear colour } \\
\hline & Healthy & Cataract & Healthy & Cataract \\
\hline $0.1-0.9$ & $30(54.6 \%)$ & 0 & $19(34.6 \%)$ & 0 \\
\hline $1.0-1.9$ & $23(41.8 \%)$ & $2(3.5 \%)$ & $22(40.0 \%)$ & 0 \\
\hline $2.0-2.9$ & $2(3.6 \%)$ & $13(22.4 \%)$ & $13(23.6 \%)$ & $15(25.9 \%)$ \\
\hline $3.0-3.9$ & 0 & $19(32.8 \%)$ & $1(1.8 \%)$ & $21(36.2 \%)$ \\
\hline $4.0-4.9$ & 0 & $15(25.9 \%)$ & 0 & $15(25.9 \%)$ \\
\hline $5.0-5.9$ & 0 & $5(8.6 \%)$ & 0 & $2(3.5 \%)$ \\
\hline $6.0-6.9$ & 0 & $4(6.9 \%)$ & 0 & $5(8.6 \%)$ \\
\hline All & $55(100 \%)$ & $58(100 \%)$ & $55(100 \%)$ & $58(100 \%)$ \\
\hline
\end{tabular}

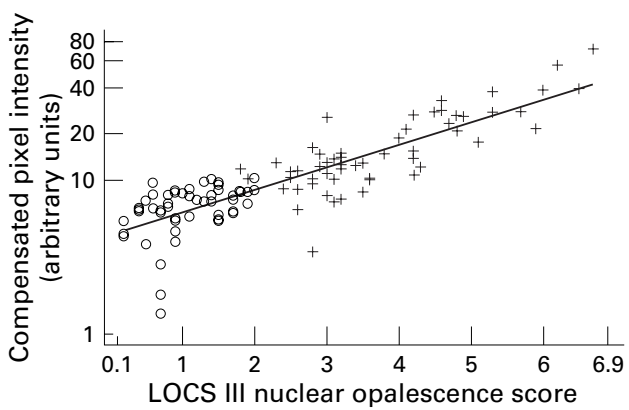

Figure 4 Relation between compensated pixel intensities (laser slit lamp) and LOCS III opalescence scores in 55 eyes of healthy subjects (O), and 58 eyes of subjects from the cataract waiting list $(+)$.

dark background area of the same anterior segment image (Fig 3).

The study was approved by the Southampton and South West Hampshire Health Authority and University of Southampton joint ethics committee. Written informed consent was sought and obtained from all participants.

\section{STATISTICAL METHODS}

Compensated pixel intensities were calculated as the mean pixel intensity in the region of interest minus the mean pixel intensity in the background region.

Reproducibility coefficients (95\% range for the difference between two repeat measurements on the same eye) were used as a measure of the absolute reproducibility of each method of cataract grading. This is only an appropriate measure if the variability does not depend on the value of the measurement ${ }^{11}$; plots of the difference between the two repeat measurements against the mean of the two were used to check this assumption.

Intraclass correlation coefficients estimate the proportion of total variability due to differences between subjects (or eyes in the case of this study). ${ }^{12}$ The reproducibility coefficient cannot be used for comparing the reproducibility of the two methods because they use different scales of measurement. ${ }^{13}$ Since the main focus of the study was to assess the ability of the laser slit lamp method to provide a reproducible measure of cataract, intraclass correlations were calculated separately for healthy and cataract subjects. The distribution of compensated pixel intensities was positively skewed. However, more crucial to this analysis was the assumption that the variability was constant; this was true only on the original (untransformed) scale, thus the untransformed data were used in most of the analysis. Descriptive analyses were carried out on the first measurements of each eye.

\section{Results}

The distribution of LOCS III gradings for nuclear opalescence and colour (based on the consensus score) for the first lens photographs is tabulated according to whether the subject was from the cataract waiting list (cataract) or from the research institution (healthy) in Table 1 . The median interval between repeat measurements was 3 days (minimum 1 day, maximum 29 days). Nuclear opalescence grades varied from 0.2 to 2.0 in the eyes of healthy subjects, and from 1.8 to 6.7 in the eyes of cataract subjects. The corresponding ranges for nuclear colour were from 0.2 to 3.0 for healthy subjects and from 2.4 to 6.5 for cataract subjects. The median pixel intensity (first measurement) was 6.8 in the eyes of healthy subjects (range 1.3-10.2) and 13.4 in the cataract patients' eyes (range 3.4-72). Pixel intensities were plotted against LOCS III scores for all eyes to assess the validity of the laser slit lamp method (Fig 4). The graph shows that there is a linear, increasing relation between the logged pixel intensities and the LOCS III opalescence scores and that they are fairly closely correlated; the Pearson correlation coefficient was $0.85(\mathrm{p}<0.001)$.

The relation between the lens opacity scores obtained from the two visits is shown for the laser slit lamp in Figure 5A and for LOCS III in Figure 6A. These plots indicate that both
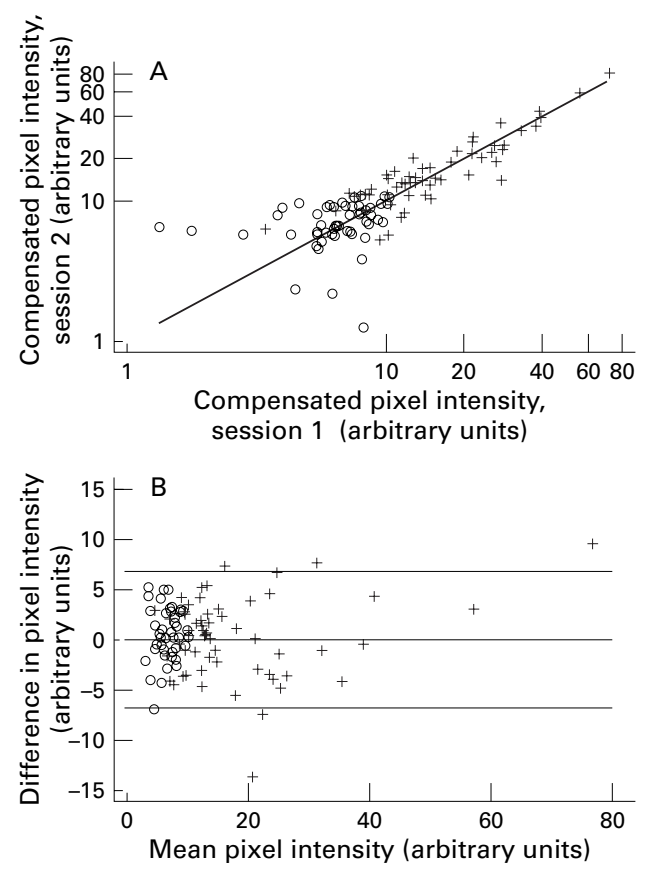

Figure 5 (A) Relation between compensated pixel intensities (laser slit lamp) measured at the first and second visits for 55 eyes of healthy subjects $(O)$ and 58 eyes of subjects from the cataract waiting list (+). (B)

Bland-Altman plot for the laser slit lamp. Difference between the two repeat compensated pixel intensities plotted against the mean of the two readings for 55 eyes of healthy subjects (O) and 58 eyes of subjects from the cataract waiting list $(+)$. 

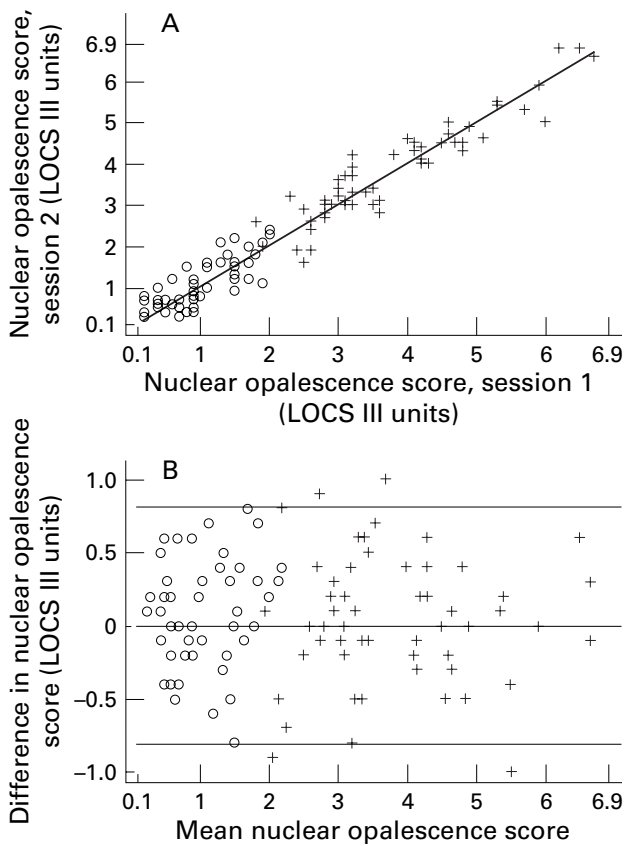

(LOCS III units)

Figure 6 (A) Relation between LOCS III nuclear opalescence scores measured at the first and second visits for 55 eyes of healthy subjects (O) and 58 eyes of subjects from the cataract waiting list (+). (B) Bland-Altman plot for LOCS III nuclear opalescence. Difference between LOCS III nuclear opalescence scores for the two visits plotted against the mean for 55 eyes of healthy subjects $(0)$ and 58 eyes of subjects from the cataract waiting list $(+)$.

methods appear to be fairly repeatable, with all points lying on or very close to the line of identity. For both the laser slit lamp and LOCS III, the mean difference between the first and second measurements did not differ significantly from zero (actual values given in Table 2). The reproducibility is indicated more formally by the Bland-Altman plots in Figures $5 \mathrm{~B}$ and $6 \mathrm{~B}$ (showing the differences in lens opacity score between the two visits plotted against the mean).

For the laser slit lamp $95 \%$ of repeat measurements were within 6.8 compensated pixel units of each other. For LOCS III, 95\% of repeat measurements were within 0.77 (nuclear colour) and 0.81 (nuclear opalescence) LOCS III units of each other.

Table 2 gives the intraclass correlations for both grading methods. The overall intraclass correlation was $95 \%$ (95\% CI $92.9 \%$ to $96.5 \%$ ) for the laser slit lamp and 97\% (95\% CI 95.6\% to $97.9 \%$ ) for LOCS III nuclear opalescence. For cataract subjects analysed separately, the intraclass correlation was $94.7 \% \quad(95 \%$ CI

Table 2 Mean difference between repeat measurements, repeatibility coefficients, and intraclass correlation coefficients for each method of cataract grading. (Calculated on 113 eyes, each measured twice)

\begin{tabular}{|c|c|c|c|}
\hline & \multirow{2}{*}{$\frac{\text { Laser slit lamp }}{\text { pixel intensity }}$} & \multicolumn{2}{|l|}{ LOCS III score } \\
\hline & & Nuclear colour & Nuclear opalescence \\
\hline $\begin{array}{r}\text { Mean difference (session } \\
2 \text {-session 1) }(95 \% \mathrm{CI})\end{array}$ & $\begin{array}{l}0.40 \\
(-0.24-1.04)\end{array}$ & $\begin{array}{l}0.06 \\
(-0.01-0.13)\end{array}$ & $0.06(-0.01-0.14)$ \\
\hline $\begin{array}{l}\text { Intraclass correlation }(\%) \\
\quad(95 \% \mathrm{CI})\end{array}$ & & & \\
\hline Healthy subjects & $38.6(12.9-59.2)$ & $82.3(71.1-89.4)$ & $76.1(62.3-85.4)$ \\
\hline Cataract subjects & $94.7(91.2-96.9)$ & $94.1(90.2-96.4)$ & $93.5(89.4-96.1)$ \\
\hline All subjects & $95.0(92.9-96.5)$ & 96.7 (95.3-97.8) & $97.0(95.6-97.9)$ \\
\hline
\end{tabular}

$91.2 \%$ to $96.9 \%$ ) for the laser slit lamp, and $93.5 \%$ (95\% CI $89.4 \%$ to $96.1 \%$ ) for LOCS III nuclear opalescence. Among healthy subjects, the intraclass correlation was $38.6 \%$ (95\% CI $12.9 \%$ to $59.2 \%$ ) for the laser slit lamp and $76.1 \%$ (95\% CI $62.3 \%$ to $85.4 \%$ ) for LOCS III nuclear opalescence. The values of intraclass correlation for nuclear colour are similar to those for nuclear opalescence (Table 2).

\section{Discussion}

We have shown a linear relation between lens opacity as assessed by the compensated pixel intensities obtained from the laser slit lamp and the consensual LOCS III scores for nuclear opalescence. Furthermore the reproducibility of the laser slit lamp is high, and similar to values we obtained using LOCS III. Intraclass correlation coefficients were $95 \%$ and $97 \%$ for the laser slit lamp and LOCS III nuclear opalescence respectively and did not differ significantly. Our data concerning the validity of the laser slit lamp data are similar to the results of other investigators who compared scores obtained by clinical grading with a digital method using Scheimpflug photography. ${ }^{14}$ Our data concerning the reproducibility of LOCS III agreed with the results of other researchers, ${ }^{15}$ who reported an intraclass correlation of $97 \%$ for LOCS III nuclear opalescence using photograding. These figures are also similar to values reported by the developers of LOCS III $-96 \%,{ }^{16}$ and $95 \%,{ }^{17}$ for grading nuclear opalescence.

When we examined the intraclass correlation for healthy volunteers and cataract subjects separately, we found that both systems were less reproducible for the healthy group. The performance of the laser slit lamp was particularly poor in this group. This is in part the result of the narrower distribution of values in healthy subjects for both systems. It may also be because neither system functions as well at the lower end of the scale. In the case of the laser slit lamp, our impression was that the values we recorded at the lower end showed a low signal to noise ratio. An improvement in the performance of the system might be achieved by recording a neutral and stable background device (for example, Ulbricht sphere) for calibration and use in subtractive calculations.

Another measurement device that has been developed to quantify cataract objectively is the lens opacity meter. It measures the amount of stray light produced by the lens in response to a modulated beam of incident red light. ${ }^{18}$ The instrument was developed in order to estimate the influence of cataract on subjective measures of visual function, such as visual field testing. "Opacity value" readings produced by the lens opacity meter correlated positively with density values from the nucleus as measured by Scheimpflug photography. ${ }^{19}$ However, all stray light produced anywhere between cornea and the posterior capsule of the lens is detected, ${ }^{18}$ and the instrument cannot give detailed information about different scattering properties of the various regions of the lens. ${ }^{20}$ 
Objective methods of nuclear cataract quantification have otherwise incorporated Scheimpflug optics in the imaging system. We challenge this requirement. Intraclass correlations for one such instrument ${ }^{15}$ ranged from $95 \%$ to $98 \%$ which is similar to the values we have obtained for the laser slit lamp.

In summary, we have demonstrated that the laser slit lamp measure of nuclear lens opacity correlated positively with values obtained using a well recognised system of grading-LOCS III. We have also shown the system to be similar in reproducibility of grading nuclear cataract to LOCS III and to more expensive objective systems for measuring nuclear cataract. However, the laser slit lamp has poor reproducibility among subjects without significant lens opacity. This is probably because of the lower levels of reflected light from clear lenses. Further development of the system may enhance its performance.

\section{Appendix}

DESCRIPTION OF THE LASER SLIT LAMP

A low power diode laser housed in a vertical column replaced the illumination arm of a Zeiss slit lamp. The optics of the laser were modified by placing a high power convex cylindrical lens in the path of the beam and then directing the beam against a 45 degree mirror in order to produce a vertical slit of light. The column can be rotated about a concentric vertical axis so that a vertical slit of light can be projected into the eye in similar fashion to a conventional slit lamp. The brightness of the beam was attenuated by positioning a vertical slit aperture in the path of the beam. The laser wavelength was $635 \mathrm{~nm}$ and the power of the whole attenuated beam was measured as 143 $\mu \mathrm{W}$ using the Newport optical power meter Model 840 . This gave a maximum permissible exposure time to the eye of 8.5 minutes. ${ }^{21}$ This comfortably exceeds the time necessary to acquire an image of the lens. However, only the central portion of the vertical laser slit is actually used to make measurements; this increases the safety margin still further. Using an artificial pupil of $7 \mathrm{~mm}$ diameter, we measured the power of the beam actually entering the eye to be $36 \mu \mathrm{W}$, which gave a maximum permitted exposure time of 34 minutes. Because the laser light cannot, in fact, be focused on to the retina during examination, there is an appreciable additional safety margin beyond even this time.
We used a Carl Zeiss beam splitter coupled to a COHU monochrome video camera and PC with a Chameleon video card to display and capture live video images. We used Image Pro software to analyse the image files which were stored in .tiff format. Philip Lempert designed and built the laser slit lamp and has filed a patent for the design. All measurements and analyses were carried out independently by the other authors none of whom has a proprietary interest in the instrument.

Dr Hall was supported by a Wellcome Trust research training fellowship in clinical epidemiology. Miss Susan Beare and Mr Stephen Smith assisted with data collection and processing. Mr Tony Murrills and Miss Angela Cotton of the Department of Medical Physics and Bioengineering, Southampton General Hospital, measured the power of the laser and calculated maximum permissible exposure times for the eye. Mr Frank Clewlow and permissible exposure times for the eye. Mr Frank Clewlow mechanical assistance with the equipment.

1 Brown NAP, Bron AJ, Ayliffe W, et al. The objective assessment of cataract. Eye 1987;1:234-46.

2 Mehra V, Minassian DC. A rapid method of grading cataract in epidemiological studies and eye surveys. $\mathrm{Br} \mathscr{f}$ Ophthalmol 1988;72:801-3.

3 Laties A, Keates E, Lippa E, et al. Field test reliability of a new lens opacity rating system utilizing slit-lamp examination. Lens Eye Tox Res 1989;6:443-64.

4 Sparrow JM, Ayliffe W, Bron AJ, et al. Inter-observer and intra-observer variability of the Oxford clinical cataract classification and grading system. Int Ophthalmol 1988;11: 151-7.

5 Chylack LT, Wolfe JK, Singer DM, et al. The lens opacities classification system III. Arch Ophthalmol 1993;111:831-6. 6 Christen WG, Manson JE, Seddon JM, et al. A prospective study of cigarette smoking and risk of cataract in men. FAMA 1992;268:989-93.

7 Taylor HR, West SK, Rosenthal FS, et al. Effect of ultraviolet radiation on cataract formation. $N \mathrm{Engl} \mathcal{F} \mathrm{Med}$ 1988;319:1429-33

8 Sasaki K, Fujisawa K, Sakamoto Y. Quantitative evaluation of nuclear cataract using image analysis. Ophthalmic Res 1992;24(suppl 1):26-31.

9 Lempert P. A new instrument for objective density measurement of the crystalline lens nucleus: correlation of visual acuity with nuclear density. In: Parel JM, ed. Proceedings of the International Society for Optical Engineering 1992; ings of the Inter

10 Image Pro Plus (2.0). Silver Springs, MD: Media Cybernetics, Inc, 1995.

11 Chinn S. The assessment of methods of measurement. Stat Med 1990;9:351-62.

2 Snedecor GW, Cochran WG. One-way classifications. Analysis of variance. In: Statistical methods. 6th ed. Ames: Iowa State University Press, 1967:258-96.

13 Chinn S. Repeatability and method comparison. Thorax 1991;46:454-6.

14 Robman LD, McCarty CA, Garrett SKM, et al. Comparison of clinical and digital assessment of nuclear optical density. Ophthalmic Res 1999;31:119-26.

15 Chylack LT, Wolfe JK, Friend J, et al. Validation of methods for the assessment of cataract progression in the Roche European-American Anticataract Trial (REACT). Ophthalmic Epidemiol 1995;2:59-75.

16 Karbassi M, Khu PM, Singer DM, et al. Evaluation of Lens Opacities Classification System III applied at the slitlamp. Opacities Classification System
Optom Vis Sci 1993;70:923-8.

17 Khu PM, Karbassi M, Singer DM, et al. Effect of cataract type and severity on lens grading. Ophthalmic Res 1994;26(suppl 1):61-7.

18 Flammer J, Bebie H. lens opacity meter:a new instrument to quantify lens opacity. Ophthalmologica, Basel 1987;195:6972.

19 Mizuno T, Sasaki K, Sakamoto Y. Evaluation of nuclear opacification using a lens opacity meter. Ophthalmic Res 1990;22(suppl 1):36-40.

20 Brown NAP, Bron AJ, Sparrow J. Methods for evaluation of lens changes. Int Ophthalmol 1988;12:229-35.

21 European Committee for Electrotechnical Standardisation. Safety of laser products. Part 1. Equipment classification, requirements and user's guide. BS EN 60825-1. London: British Standards Institution, 1994. 